\title{
Das Bodenwertmodell für die Grundsteuer: Unzulänglichkeiten und mögliche Alternativen
}

\begin{abstract}
2018 wurde das bisherige Verfahren der Grundsteuererhebung als verfassungswidrig erklärt. Den Bundesländern ist bis Ende 2024 Zeit für eine Neukonzeption der Grundsteuer eingeräumt worden. Baden-Württemberg hat eine Vorreiterrolle eingenommen und sich auf ein Bodenwertmodell festgelegt, das aber Ungleichbehandlungen nicht vermeiden kann und Gefahr läuft, als verfassungswidrig eingeordnet zu werden. Entsprechendes gilt auch für das Flächenmodell. Eine Alternative könnte eine Beteiligung der Gemeinden an der Einkommensund Umsatzsteuer sein.
\end{abstract}

Das Bundesverfassungsgericht (2018) hatte mit seinem Urteil vom 10. April 2018 die Grundsteuererhebung als verfassungswidrig bewertet. Zugleich wurde der Gesetzgeber verpflichtet, „eine Neuregelung spätestens bis zum 31. Dezember 2019 zu treffen“ (Bundesverfassungsgericht 2018, Satz 2). Dem folgte eine längere Diskussion über mögliche Vorgehensweisen zwischen Bund und Ländern. Bis schließlich mit dem Gesetz zur Änderung des Grundgesetzes (Art. 72, 105 und 125b) vom 15. November 2019 (BGBI, 2019) festgehalten wurde, dass ein einheitliches Bundesgesetz für die Grundsteuer nicht vorgesehen ist. Der Bund hat sich auf die konkurrierende Gesetzgebung über die Grundsteuer beschränkt (Art. 105 Abs. 2 GG). Von erheblicher Bedeutung für die Bürger:innen und Steuerzahlenden ist aber der in Art. 125b angefügte Abs. 3, wonach ab dem 1. Januar 2025 abweichendes Landesrecht der Erhebung der Grundsteuer zugrunde gelegt werden darf. Damit wird zumindest für den engeren Bereich der Grundsteuerzahllasten in den Bundesländern das Gebot der „gleichwertigen Lebensverhältnisse“ (Art. 72 Abs. 2 GG) aufgegeben.

Mit dem Gesetz zur Regelung einer Landesgrundsteuer (Landesgrundsteuergesetz - LGrStG) vom 4. November 2020 (GBI 2020, 974 ff.) hat Baden-Württemberg die Initiative für eine Neukonzeption des Grundsteuerrechts ergriffen und eine Bodenwertsteuer eingeführt. Das „Gesetz soll eine einfache, transparente und gut administrierbare Grundsteuer für Baden-Württemberg ermöglichen. [...] Der Entwurf ist verfassungsfest [...]. [...] Neben der Leistungsfähigkeit wird auch der Äquivalenzgedanke explizit als Belastungsgrund genannt“ (Landtag von Baden-Württemberg, 2020, 97). „Die

(C) Der/die Autor:in(nen) 2021. Open Access: Dieser Artikel wird unter der Creative Commons Namensnennung 4.0 International Lizenz veröffentlicht (creativecommons.org/licenses/by/4.0/deed.de).

Open Access wird durch die ZBW - Leibniz-Informationszentrum Wirtschaft gefördert.
Landesgrundsteuer basiert auf einem vollkommen neuen System, welches das Gebäude ausblendet und sich im Rahmen der Bewertung ausschließlich auf den Grund und Boden bezieht" (Landtag von Baden-Württemberg, 2020, 98). „Der Grundsteuerwert der Grundstücke ermittelt sich durch Multiplikation ihrer Fläche des Grund und Bodens mit dem jeweiligen Bodenrichtwert gemäß $\$ 196$ des Baugesetzbuchs [...]. [...] Die Bodenrichtwerte sind von den Gutachterausschüssen [...] auf den Hauptfeststellungszeitpunkt zu ermitteln [...] und an die zuständigen Finanzbehörden zu übermitteln“ (§38 Abs. 1 und 2 LGrStG). „Auf den 1. Januar 2025 findet eine Hauptveranlagung der Grundsteuermessbeträge statt (Hauptveranlagung, 2025)“ (§59 Abs. 1 LGrStG).

\section{Kritik am Bodenwertmodell}

Das Bodenwertmodell greift aus der Fülle der wertprägenden Eigenschaften eines Grundstücks eine einzelne Komponente heraus, die im Vergleich der Werte unterschiedlicher Grundbesitzobjekte durchaus wichtig, aber nicht unbedingt zentral und in der Regel sogar nachrangig ist, d.h. unter $50 \%$ des Grundstückwerts einschließlich der Aufbauten liegt. Der Bodenwert muss sich auch nicht zwischen unterschiedlichen Grundstücken über die Zeit hin, d.h. auch

Prof. Dr. Gerhard Graf war Professor für Volkswirtschaftslehre und Finanzwissenschaft an der Verwaltungsfachhochschule in Wiesbaden sowie Privatdozent an der Johannes Gutenberg-Universität Mainz und Lehrbeauftragter für International Economics und Public Finance an der European Business School in Oestrich-Winkel. 
zwischen möglichen Hauptfeststellungszeitpunkten, parallel oder verzerrungsfrei entwickeln. Damit ist zumindest die Gefahr gegeben, dass das Gleichheitsgebot (Art. 3 GG) verletzt ist. Daraus folgt eine mangelnde Berücksichtigung des Leistungsfähigkeitsprinzips zwischen mehreren Grundstücken. Die apodiktische Feststellung in der Gesetzesbegründung, dass der „Entwurf verfassungsfest“ (Landtag von Baden-Württemberg, 2020, 97) sei, kann nicht nachvollzogen werden und wird auch keinen Bestand haben. ${ }^{1}$

Das Bodenwertmodell will mit der Erfassung einer Wertgröße das Resultat übernehmen, das im Rahmen einer Marktwirtschaft in einer gesellschaftlichen Einschätzung für Grundstücke zustande kommt. Insoweit spiegelt der Bodenwert in seinen Änderungen allgemeine wirtschaftliche Entwicklungen wider. Er kann steigen oder fallen und er soll die divergierenden Einflüsse auf unterschiedliche Grundstücke erfassen. Von daher erscheint das Bodenwertmodell zunächst als ein besonders geeignetes Modell, um die Vielzahl der in Baden-Württemberg belegenen Steuerobjekte, rund 5,6 Mio., verhältnismäßig einfach oder gut administrierbar in ein neues System zu integrieren.

Allerdings darf nicht verkannt werden, dass der Bodenwert neben der von vornherein eingeschränkten Sicht auf die Wertverhältnisse eines Grundstücks, einschließlich der Werte der Immobilien, nicht immer hinreichend genau sein wird, um Vergleichen zwischen unterschiedlichen Grundstücken zweifelsfrei standzuhalten. Es ist daher mit Streitfällen zu rechnen. Die mangelnde Genauigkeit und die Streitanfälligkeit werden zudem zunehmen, da die Grundsteuerwerte nur ,in Zeitabständen von je sieben Jahren allgemein festgestellt“ (§15 Abs. 1 LGrStG) werden. „Der Hauptfeststellung werden die Verhältnisse zu Beginn des Kalenderjahres (Hauptfeststellungszeitpunkt) zugrunde gelegt“ (§15 Abs. 2 LGrStG). Die Gesetzesformulierungen belegen die inhärente zeitliche Nachläufigkeit der Bodenbewertung und die daraus folgenden Grundsteuerwerte. Dies wird auch zu EinbuBen bei der Gleichartigkeit der Bewertung führen, denn die Werte des Bodens und der Grundstücke insgesamt bewegen sich in einem zeitlichen Kontinuum. Eine Stichtagsbewertung kann dem nur mit Abstrichen folgen.

Das Bodenwertmodell wird zudem beim Vergleich von Grundstücken und deren Wertverhältnissen immer wieder mit dem Problem behaftet sein, dass die Werte bebauter

1 Die Ausführungen konzentrieren sich auf die Grundsteuer B. Die meisten Argumente sind auch für die Grundsteuer A gültig. Diese ist im o.a. Landesgrundsteuergesetz behandelt. Das Gesetz widmet sich dem land- und forstwirtschaftlichen Vermögen in den $\S \S 26-36$, zu denen neun Anlagen hinzugefügt sind. Dies darf durchaus als Beispiel gewertet werden, wie eine Obrigkeit kleinteilige Regelungen für einen Wirtschaftsbereich vorsieht, aus dem kaum finanzielle Erträge erzielt werden, dem aber mit einer Fülle von Einzelfalllösungen scheinbar individuelle Gerechtigkeit widerfahren soll.
Grundstücke Entwicklungen annehmen, die mit dem Bodenwert nicht synchron gehen und gegebenenfalls weit davon abweichen. Dann stellt sich für die Steuerzahlenden die Frage der Gleichbehandlung. Die Problematik der divergierenden Wertentwicklungen von Vermögensgegenständen und deren Bestandteilen gilt natürlich für alle auf einen stichtagsbezogenen Wert rekurrierenden Bemessungsgrundlagen von Steuerfestsetzungen. Dies hat die alte Grundsteuerregelung zu Fall gebracht. Über die Zeit wird die Bodenwertsteuer dieser Gefahr nicht entgehen können.

Schließlich darf bei dem Bodenwertmodell nicht verkannt werden, dass seine zur Steuerbemessung erforderliche Umsetzung für die Kalkulation der Steuermesswerte nicht ohne Aufwand geschieht. Dieser Aufwand wird zunehmen, wenn es für die Grundstückseigentümer:innen um die Festsetzung der Steuerzahllasten geht. Dabei werden die von der Finanzverwaltung benutzten Bodenrichtwerte bzw. ihre Änderungen und Aktualisierungen (z. B. nach sieben Jahren) nicht ohne Widerspruch bleiben. Einsprüche bis hin zu Gerichtsverfahren sind die mögliche Folge.

Einen besonderen Nachteil des Bodenwertmodells sieht Kirchhof (2020) in der bestehenden Ungleichbehandlung von bebauten und unbebauten Grundstücken. Nach dem in Baden-Württemberg präferierten Bodenwertmodell werden Gebäude nicht erfasst und nicht bewertet. Die Steuer soll damit „für unbebaute und vergleichbare bebaute Grundstücke“ (Kirchhof, 2020, 44) gleich sein. Die Steuer will die Eigentümer:innen insoweit drängen, Immobilien zu errichten, um die Wohnungsnot in Ballungszentren zu lindern (Kirchhoff, 2020). Kirchhof (2020) wendet sich explizit gegen die mit der Bodenwertsteuer beabsichtigte Lenkungswirkung, die darin besteht, dass unbebaute Grundstücke mit bebauten gleichgestellt werden, um so Druck auf den Grundstückseigentümer auszuüben, das Grundstück zu bebauen und einen entsprechenden Ertrag aus dem Grundstück zu ziehen. Er hält die Bodenwertsteuer aus diesem Grund für verfassungswidrig.

\section{Das Flächenmodell als Alternative?}

Das Flächenmodell - eventuell „veredelt“ um einen Lagefaktor - wird vorrangig damit begründet, einen einfachen Maßstab für die Grundstücke benutzen zu können, der zudem nicht mit sich verändernden Bemessungsgrundlagen belastet ist. Steuerzahlende und die Kommunen könnten sich langfristig an den gefundenen Bemessungen und den Lasten der Steuerzahlungen ausrichten (Kirchhof, 2020, 47). Ganz entschieden ist Kirchhof zu widersprechen, wenn er das Flächenmodell „als Beispiel für ein modernes Steuergesetz" (Kirchof, 2020, 57) bezeichnet. Die Grundsteuer wäre mit der Bodenfläche als Bemessungsgrundlage keine moderne Steuer, sondern ein Rückfall in die Zeiten vor Hun- 
derten Jahren. Damals gab es noch keine wertabhängigen Bemessungsgrundlagen, z. B. Einkommen und Umsätze, und die Obrigkeit musste sich an Realien, wie der Größe eines Grundstücks, ausrichten, um den Zehnten (eine an der Erntemenge festgelegte reale Größe) als Abgabe zu erheben. Die Grundsteuer hat ihre tradierte Rolle als Realsteuer zumindest in der Vorstellung vieler Beobachtender noch nicht verloren, was sich auch deutlich in der recht misslichen Diskussion um neue Modelle zeigt, die die verfassungswidrig gewordene alte Konstruktion ersetzen sollen.

Ein Flächenmodell benutzt einen Teilaspekt eines Grundstücks, die Fläche, dem jedoch noch mehr als beim Bodenwertmodell die Gleichbehandlung von Grundvermögen abgeht. Realien wie Grundstücke lassen sich durchaus anhand von Flächenmaßen vergleichen. Eine steuerliche Vergleichbarkeit muss jedoch auf einer Vergleichbarkeit von Werten oder Steuerzahllasten aufbauen und darf sich nicht auf physikalische Größen beziehen. Der jeweilige Wert hat zudem den Wert nicht nur zu einem Stichtag abzubilden, sondern über die Zeit. Gerade die Inkonsistenz über die Zeit hat zu der Verfassungswidrigkeit der alten Grundsteuer geführt. Werte verändern sich dynamisch und unterliegen gesellschaftlichen und ökonomischen Einflüssen. Die Wertentwicklung wird zwischen den 36 Mio. Steuerobjekten auch nie synchron verlaufen und lässt sich daher nicht mit einem Mengenkonzept abbilden. Das Flächenmodell ist somit ungeeignet als Ersatz der einheitswertabhängigen Grundsteuer.

\section{Die Bedeutung der Einnahmen aus der Grundsteuer}

Ehe weitere Alternativen zur bisherigen Grundsteuer beleuchtet und kritisch kommentiert werden, sollte Klarheit über die finanzielle Dimension der Grundsteuereinnahmen für die Kommunalfinanzen bestehen. Hier trifft man in der wissenschaftlichen Diskussion teilweise auf Formulierungen oder Faktenbehauptungen, die völlig am Sachverhalt vorbeigehen. Als mustergültig kann erneut Kirchhof mit seinem mehrfach zitierten Gutachten „Der Belastungsgrund von Steuern - zum verfassungsrechtlichen Auftrag, die Grundsteuer zu reformieren“ (Kirchhof, 2020) gelten. „Die Grundsteuer ist eine zentrale Quelle der Gemeindefinanzierung.“ Ohne Neuregelung des Grundsteuerrechts „[...] geraten die finanziellen Grundlagen der Kommunen in Höhe von jährlich rund 14 Mrd. Euro in Gefahr" (Kirchhof, 2020, 3).

Zutreffend ist die genannte Höhe der Grundsteuereinnahmen pro Jahr von mittlerweile über 14 Mrd. Euro (2019) (Bundesbank, 2021, 61*) für alle Kommunen in Deutschland. Die Frage ist, ob sich hinter diesem Geldbetrag eine finanzielle Grundlage der Kommunen verbirgt. Achtet man nur auf das Gewicht der Grundsteuer an den kommunalen Steuereinnahmen von 71,7 Mrd. Euro in 2019 (Bundesbank, 2021), so mag dies als Beleg für deren Bedeutung (20\% der Steuereinnahmen) angesehen werden. Daneben ist aber zu berücksichtigen, dass die Gesamteinnahmen der Kommunen 2019 bei 282,3 Mrd. Euro (Statistisches Bundesamt, 2020) lagen. Das Gewicht der Grundsteuer an den Gesamteinnahmen beträgt aber nur 5,1\%. Die Grundsteuer kann insoweit nicht als besonders bedeutsame Einnahmequelle der Kommunen gewertet werden. Hinzu kommt, dass die Steuereinnahmen der Kommunen keinesfalls in erster Linie aus selbstbestimmten Steuern (mit eigenem Hebesatz oder vor Ort bestimmtem Steuersatz) bestehen, sondern sich zu einem großen Teil aus Zuweisungen von Bund und dem jeweiligen Land ergeben. Nicht zu vernachlässigen ist zudem, dass über 50 Mrd. Euro der kommunalen Steuereinnahmen Beteiligungen an gemeinschaftlichen Steuern sind, hierzu zählen der Lohnsteueranteil (33 Mrd. Euro 2019), der Anteil aus der veranlagten Einkommensteuer (9,6 Mrd. Euro), die Beteiligung an der Abgeltungssteuer (0,6 Mrd. Euro) sowie die Beteiligung an den Umsatzsteuern (8,3 Mrd. Euro) (BMF, 2020). Sollten auch die Einnahmen, die bisher aus der Grundsteuer fließen, in Form einer Zuweisung aus den großen Wertsteuern, der Einkommensteuer und der Umsatzsteuer, zusammengesetzt werden, wären lediglich die genannten Positionen aufzustocken. Als Ergebnis käme es zu einer Zuweisungssumme von insgesamt etwa 65 Mrd. (gemessen an den Werten von 2019) an die Kommunen, ohne hierfür 36 Mio. steuerliche Sachverhalte für eine relativ kleinere Einnahmenposition neu zu konstruieren und berechnen zu müssen.

\section{Mögliche Beteiligungslösung als Ersatz?}

Zwei Problembereiche sind zu prüfen und im Interesse einer solch einfachen Beteiligungslösung zufriedenstellend zu beantworten. Zum einen wäre die Frage der Eigenständigkeit der Kommunen bei ihrer Steuerbelastung für die Bürger:innen zu klären. Gehen nicht wesentliche Eigenheiten der kommunalen Selbstverwaltung unter, wenn die Kommunen nur eine Beteiligung an anderen Steuern, z.B. an Bundes- oder Gemeinschaftsteuern erhalten und die Höhe des Hebesatzes nicht mehr selbst bestimmen können? Die weitere Frage besteht in den Wirkungen für die Grundsteuerzahlenden, wenn die bisherige Grundsteuererhebung wegfällt und dafür im Wege der Kompensation die großen Wertsteuern eventuell angepasst werden müssen, um das Mehraufkommen für die erhöhte Umlage oder die Beteiligungsfinanzierung der Kommunen sicherzustellen.

Die erste Frage betrifft das Selbstverwaltungsrecht der Kommunen. Sie haben nach Art. 28 Abs. 2 GG das Recht, „alle Angelegenheiten der örtlichen Gemeinschaft im Rahmen der Gesetze in eigener Verantwortung zu regeln." „Die Gewährleistung der Selbstverwaltung umfaßt auch die Grundlagen der finanziellen Eigenverantwortung; zu diesen Grundlagen gehört eine den Gemeinden mit Hebesatzrecht 
zustehende wirtschaftskraftbezogene Steuerquelle." Das Erfordernis des zuletzt zitierten Satzes wäre auch dann erfüllt, wenn die Kommunen lediglich eine einzige Steuer mit Hebesatzrecht hätten, z. B. die Gewerbesteuer.

Allerdings ergäbe sich dann noch eine fehlende Übereinstimmung mit dem Art. 106 Abs. 6 GG. Danach steht den Gemeinden das Aufkommen der Grundsteuer und Gewerbesteuer zu (Art. 106. Abs. 1 Satz 1 GG). Darüber hinaus ist den Gemeinden das Recht einzuräumen, „die Hebesätze der Grundsteuer und Gewerbesteuer im Rahmen der Gesetze festzulegen“ (Art. 106 Abs. 6 Satz 2 GG). Gemäß Art. 106 Abs. 1 Satz 6 GG ist aber auch vorgesehen, dass das Aufkommen von Grundsteuer und Gewerbesteuer sowie der Gemeindeanteil der Einkommensteuer und der Umsatzsteuer als Bemessungsgrundlagen für Umlagen zugrunde gelegt werden können. Diese Vorgaben lassen sich nicht einfach mechanisch für ein Steuersystem ohne Grundsteuer übertragen. Sie könnten jedoch durch eine Neufassung des Art. 106 GG geheilt werden, bei der die Grundsteuer keine Erwähnung mehr findet.

Die Vorgaben aus Art. 106 Abs. 1 Satz 6 wären in geeigneter Form aber bereits eine wesentliche Grundlage für eine Beteiligungslösung der Gemeinden an den großen Steuern und den dafür zu bestimmenden Bemessungsgrundlagen. Insgesamt muss mithin den Gemeinden keine besondere Einbuße an Selbstverwaltungshoheit drohen, wenn sie auf die tradierte Grundsteuer und das Aufkommen von $5 \%$ der Einnahmen verzichten sollten. Es wäre in jedem Fall aber dann eine zumindest äquivalente Zuweisung aus den großen Steuern für sie vorzusehen. Das heißt z. B., dass die Gemeinden von ihrem jeweiligen Land im Rahmen des kommunalen Finanzausgleichs so zu stellen wären, als könnten sie zumindest betragsmäßig noch über genuine Grundsteuereinnahmen verfügen. Konkret ließe sich das im Zusammenhang mit den ohnehin anstehenden jährlichen Erörterungen zwischen dem jeweiligen Bundesland und seinen Kommunen über die Details der Finanzausgleichszahlungen regeln. Insbesondere wären die Bedarfsmesszahlen entsprechend festzusetzen, um letztlich finanzielle Einbußen der Kommunen zu vermeiden. Wegen der zu ersetzenden 14 Mrd. Euro aus der Grundsteuer stehen mithin die Kommunen in keiner finanziellen Gefahr. Eine Anpassung der Schlüsselzuweisungen unter Berücksichtigung des Bedarfs und der geringeren Steuerkraftmesszahl erscheint vielmehr als Aufgabe, die in den Ländern verhältnismäßig einfach - wenn auch mit den üblichen Diskussionen zwischen den unterschiedlichen Kommunen - gelöst werden kann.

\section{Verteilungswirkungen der bisherigen Grundsteuer}

Die bisherige Grundsteuer, die von einem Einheitswert und ursprünglich vom gemeinen Wert des Grundstücks ausge- gangen ist, hat sich insbesondere durch zwei Verteilungswirkungen ausgezeichnet. Eine Verteilungswirkung folgt dem Äquivalenzprinzip. Alle Grundstückseigentümer:innen und deren Mieter:innen beteiligen sich mit den Grundsteuerzahlungen an den allgemeinen Finanzierungslasten der Kommune für die Infrastruktur. Hierbei wird grundsätzlich niemand ausgeschlossen. Individuelle Verhältnisse der Grundsteuerzahlenden werden mit wenigen Ausnahmen nicht berücksichtigt, zumal die Grundsteuer als dingliche Last auf dem Grundstück liegt.

Die weitere Verteilungswirkung ergibt sich indirekt insoweit, als kleinere oder weniger wertvolle Grundstücke (oder mit einem weniger werthaltigen Gebäude) einen niedrigeren Einheitswert aufweisen und damit eine geringere Grundsteuer zu tragen haben. Größere Grundstücke mit eventuell auch wertvolleren Bebauungen weisen höhere Einheitswerte aus und sind insofern höher belastet. Das kleine einfache Reihenhaus und die große Villa mit dem großen Grundstück mögen nur als vereinfachte Beispiele für die hier intendierte Art der Argumentation dienen. Ähnliches gilt selbstverständlich auch beim Vergleich der kleinen 2-Zimmer-Wohnung in einem größeren Wohnblock mit dem 5-Zimmer-Penthouse in Hang- und Aussichtslage. Bereits im jetzigen System tragen die weniger werthaltigen Grundstücke eine geringere Grundsteuer als die größeren und werthaltigeren. Dies reflektiert die unterschiedliche finanzielle Leistungsfähigkeit der betroffenen Wohnungsinhaber:innen oder Grundstückseigentümer:innen.

Die bisherige Grundsteuer legt das Äquivalenz- und das Leistungsfähigkeitsprinzip zugrunde. Für beide Prinzipien gibt es jedoch keine simple mechanische Verbindung z. B. in Form eines linearen Zusammenhangs zwischen Grundstück oder Wohnung und der Höhe der Grundsteuerzahlung. Gleichwohl werden sich im Allgemeinen beide Prinzipien in der Grundsteuerschuld näherungsweise widerspiegeln.

\section{Verteilungswirkungen von Steuern}

Die bisherige Grundsteuer wird Ende 2024 in jedem Fall abgeschafft. Wie es dann um die Verteilungswirkungen der möglichen Alternativen steht, ist in der aktuellen Diskussion kaum erkennbar. Gut zu erkennen sind jedoch die Verteilungswirkungen, wenn eine Umlagelösung aufgegriffen und hierfür auf eine finanzielle Kompensation aus den großen Steuern zurückgegriffen würde. Die bisherige Grundsteuer beteiligt alle Wohnenden in einer Kommune an der Grundsteuerzahllast. Insoweit gilt die Äquivalenz. Sie beteiligt aber die Zahlenden - Eigentümer:in wie Mieter:in - nicht gleichmäßig, sondern sie führt in der Tendenz für Wohnende mit einem höheren Einkommen zu höheren Grundsteuerbeträgen. Damit zeigt sich auch das Leistungsfähigkeitsprinzip. 
Eine Beteiligungslösung an den großen Steuern (vgl. Graf, 2018) kann versuchen, die Entlastung durch den Wegfall der alten Grundsteuer zu kompensieren. Die Grundsteuerzahlenden sollten dabei nicht grundsätzlich besser aber auch nicht grundsätzlich schlechter gestellt werden als zuvor. Die Beteiligungslösung wäre daher aus Teilbeträgen der Umsatzsteuer und der Einkommensteuer zu finanzieren. Die erforderlichen Finanzsummen könnten sich aus einer leichten Erhöhung der Steuersätze bei beiden Steuerarten ergeben. Eine Erhöhung des Umsatzsteuersatzes um einen Prozentpunkt, z.B. von $19 \%$ auf $20 \%$ beim Regelsatz könnte mit einem Mehraufkommen von etwa $5 \%$ oder rund 11 Mrd. bis 12 Mrd. Euro verbunden sein. Tritt zudem im Bereich der Progressionszonen der Einkommensteuer ebenfalls eine langsame Erhöhung der Grenzsteuersätze beginnend ab 9.697 Euro (nach dem Tarif für 2021) ein, um schließlich insgesamt einen ganzen Prozentpunkt zu erreichen, würde die erste Proportionalzone des Einkommensteuertarifs (ab 57.919 Euro nach dem Tarif für 2021) mit einem Grenzsteuersatz von $43 \%$ und nicht mehr von $42 \%$ verbunden sein. Die Erhöhung der beiden Steuersätze müsste einen hinreichenden finanziellen Spielraum schaffen, um den Ausfall der bisherigen Grundsteuereinnahmen vollständig kompensieren zu können. Zugleich wäre auch das Ziel der Aufkommensneutralität, das bei gegenwärtig diskutierten Alternativen eine Wunschvorstellung bleibt, grundsätzlich gewahrt.

Fazit

Für die alte Grundsteuer gibt es durchaus machbare Alternativen, die ohne millionenfache Neuveranlagungen auskommen. Zudem wären die zu erwartenden Belastungswirkungen für die Steuerzahlenden sehr nahe bei den aktuellen Belastungswirkungen der Grundsteuer. Außerdem könnten die Steuerzahlenden insgesamt darauf vertrauen, dass die Beteiligungslösung oder Umlagelösung nicht zu beliebigen Steuererhöhungen führt, denn die Kommunen hätten keine aktiven oder gar uneingeschränkten Möglichkeiten, an den Hebesätzen der Grundsteuer zu drehen, da sie das Finan- zierungsinstrument der Grundsteuer nicht mehr eigenständig ausnutzen können. Die Finanzbedarfe der Kommunen wären im Rahmen der laufenden Verhandlungen mit dem jeweiligen Bundesland bei der Festlegung der Details zum Finanzausgleich zu regeln.

Eine Übernahme des vorgeschlagenen Beteiligungsmodells hätte wesentliche Eigenschaften, die für ein heutiges, modernes Steuersystem vorbildhaft sein können. Es wäre einfach, effizient und kostengünstig. Da außerdem keine völlig unvorhersehbaren und ungewünschten Verteilungswirkungen eintreten, wäre das Modell auch gerecht. Bekanntermaßen haben heutige politische Entscheidungsträger:innen im Zusammenhang mit ihrer Gesetzgebungsfunktion nicht immer das Ziel einfacher und allgemein gerechter Lösungen. Gleichwohl sollte angesichts der quantitativ großen Aufgabe millionenfacher Neuveranlagungen für eine im Kontext des Gesamtstaates relativ kleine Einnahme, die keinesfalls ohne sinnvolle Alternativen ist, auch eine zielführende einfache Lösung zumindest erwogen werden.

\section{Literatur}

BGBI (2019), Bundesgesetzblatt Jg. 2019, Teil I, Nr. 39, Gesetz zur Änderung des Grundgesetzes (Artikel 72, 105 und 125b).

BMF (2020), I A 6 Steuereinnahmen (ohne reine Gemeindesteuern) in Tsd. Euro - Bundesgebiet insgesamt - nach Gebietskörperschaften, Stand 06.02.2020, https://www.bundesfinanzministerium.de/ Content/DE/Standardartikel/Themen/Steuern/Steuerschaetzungen und_Steuereinnahmen/2020-04-21-steuereinnahmen-1-vierteljahr.p df;jsessionid=C3AC1B9D56B2D7707C97C50F8A058874. delivery2replication?__blob=publicationFile\&v=3 (25. Februar 2021).

Bundesbank (2021), Monatsbericht Januar 2021, 73(1).

Bundesverfassungsgericht (2018), Urteil 1-BvR 889/12 vom 10. April.

GBI (2020), Gesetzblatt für Baden-Württemberg (GBI.2020), Nr. 40.

Graf, G. (2018), Ersatz für die Grundsteuer: eine einfache, effiziente, kostengünstige und gerechte Lösung, Wirtschaftsdienst, 98(9), 669-672.

Kirchhof, G. (2020), Der Belastungsgrund von Steuern - zum verfassungsrechtlichen Auftrag, die Grundsteuer zu reformieren, Gutachten im Auftrag des ZIA Zentraler Immobilienausschuss e. V., April.

Landtag von Baden-Württemberg (2020), Drucksache 16/8907.

Statistisches Bundesamt (2020), Einnahmen des Öffentlichen Gesamthaushalts.

Title: A Property-tax Based Solely on the Value of the Plot: Deficiencies and Alternatives

Abstract: In 2018, the highest constitutional court in Germany declared that property tax is not in conformity with the constitution. The German states were given until 2024 to install a modified solution. The state of Baden-Württemberg was the first to pass a new law adopting a model of a property tax based solely on the value of the plot. This solution, however, raises again doubts about the conformity with the constitution. Similar problems are encountered if only the technical size of the plot is used as a base for a property tax. The paper proposes providing the communities with a share of the revenues of the federal income tax and the sales tax, thereby avoiding millions of new assessments for all properties.

JEL Classification: H71, K34, R51 